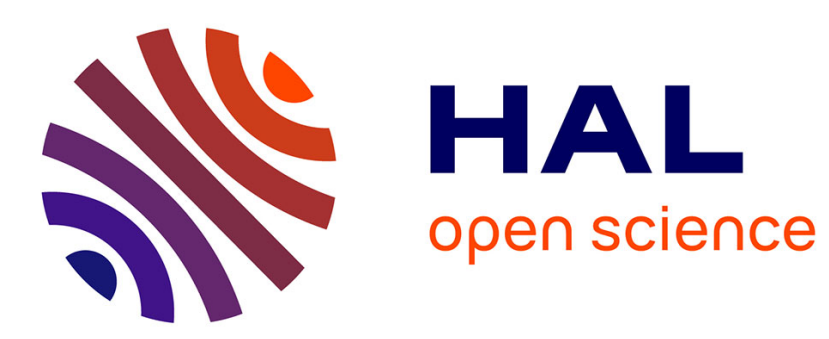

\title{
Conductivity and Transmissivity of a Single Fracture
}

\author{
V. Mourzenko, Jean-François Thovert, P. Adler
}

\section{To cite this version:}

V. Mourzenko, Jean-François Thovert, P. Adler. Conductivity and Transmissivity of a Single Fracture.

Transport in Porous Media, 2018, 123 (2), pp.235-256. 10.1007/s11242-018-1037-y . hal-02295481

\section{HAL Id: hal-02295481 \\ https://hal.science/hal-02295481}

Submitted on 29 Mar 2021

HAL is a multi-disciplinary open access archive for the deposit and dissemination of scientific research documents, whether they are published or not. The documents may come from teaching and research institutions in France or abroad, or from public or private research centers.
L'archive ouverte pluridisciplinaire HAL, est destinée au dépôt et à la diffusion de documents scientifiques de niveau recherche, publiés ou non, émanant des établissements d'enseignement et de recherche français ou étrangers, des laboratoires publics ou privés. 


\title{
Conductivity and transmissivity of a single fracture
}

\author{
2 V.V. Mourzenko · J.-F. Thovert · P.M.Adler
}

4 Received: date / Revised version: date
5 Abstract The effective flow and conduction properties of fractures with Gaussian spatial correlations are investigated by solving the microscale governing equations in threedimensional samples, along the lines initiated by $[1,2]$ but in greater details, over a wider range of the parameters, and with greatly improved accuracy. The effective transport coefficients are related to intrinsic geometrical characteristics, quantified by the mean aperture, the surface roughness RMS amplitude, its correlation length, and the intercorrelation coefficient of the roughness on the two surfaces. Extensive results are presented and analyzed. An empirical relationship between the transmissivity and conductivity is formulated, the validity of the Reynolds approximation is assessed, and heuristic expressions are proposed for the direct estimation of the transport coefficients as functions of the fracture geometrical characteristics.

Keywords Fracture $\cdot$ Conductivity $\cdot$ Permeability $\cdot$ Transmissivity

\section{Introduction}

Estimations of transport properties of single fractures are of large practical interest since the fractures control the properties of fractured media. The main purpose of this paper is to revisit the estimations of transmissivity and conductivity that were previously given by Mourzenko et al. [1] and Volik et al. [2], and to propose semi empirical or heuristic correlations which can be used for practical purposes.

Developments in theoretical and experimental studies of hydraulic behaviour of single fractures are periodically summarized; an extensive review of the early literature is provided by Adler and Thovert [3], and a more recent one by Zimmerman and Main [4].

V.V. Mourzenko

Institut P', CNRS, Bât. SP2MI, BP 30179, 86962 Futuroscope Cedex, France E-mail: mourzenko@ensma.fr J.-F. Thovert

Institut P', CNRS, Bât. SP2MI, BP 30179, 86962 Futuroscope Cedex, France E-mail: thovert @ensma.fr

P.M.Adler

UPMC METIS. Boîte 105, 4 place Jussieu, 75252 Paris cedex 05, France E-mail: pierre.adler@upmc.fr 
A lot of attention was devoted to this topic since the 1970s, but first the lubrication approximation was used almost exclusively in the so called Reynolds approximation; it consists in solving a two dimensional equation instead of the governing equations in three dimensions. The first contribution in this respect appears to be due to Patir and Cheng [5] and was followed by many others such as Zimmerman et al. [6]. Some initial contributions were concerned by the hydromechanical behaviour of fractures (Witherspoon et al. [7], Tsang and Witherspoon [8]).

It is not until the mid nineties that the Stokes equation was solved in three dimensional random fractures by [1]; Lattice Gas Automata was applied the same year to two dimensional fractures by Gutfraind and Hansen [9] and Brown et al. [10], and later to threedimensional fractures by [11] and Madadi and Sahimi [12]. [2] used the same methodology as [1] and solved the Laplace equation in three dimensions in order to calculate the macroscopic conductivity of fractures with Gaussian surfaces. Three-dimensional fractures with self-affine surfaces were addressed by Mourzenko et al. for conductivity [13] and permeability [14].

Despite these early 3D contributions, two dimensional fractures are still the topic of some studies. Some of them considered fractures with self-affine profiles (Oron and Berkowitz [15], Drazer and Koplik [16]) or real measured profiles (Koyama et al. [17]).

A method of perturbation was used by Basha and El-Asmar [18] in order to obtain the flux and pressure distribution in two-dimensional rough-walled channels and Crandall et al. [19] studied them numerically by using a finite-volume CFD package. Sisavath et al. [20] derived an expression for the hydraulic aperture of a sinusoidal fracture from Stokes equations by a perturbation method. Malevich et al. [21] performed formal expansions by an analytical and numerical algorithm for channels bounded by three dimensional wavy walls; Adler et al. [22] extended their study to inertial flows.

The method of asymptotic expansions was applied by Plouraboue et al. [23] in order to derive expressions for the conductivity and transmissivity of rough-walled fractures. Brush and Thomson [24] performed a numerical study of 3D Navier-Stokes equations in synthetic fractures and compared their solutions with those of the Reynolds equation.

Possible modifications of the Reynolds equation were considered by Ge [25], Mallikamas and Rajaram [26] and Nicholl and Detwiler [27] in order to take into account the local variations of the aperture field, i.e. to introduce the derivatives of this field directly in this equation. Corrections for local orientation and roughness effects are introduced by Wang et al. [28].

Non-linear fracture flows have also reveived some attention. For instance, Skjetne et al. [29] discussed and investigated by two-dimensional Lattice Boltzmann simulations the differences between low-Reynolds number creeping and high-Reynolds number inertial flows. Flows of non-Newtonian fluids, which are used in reservoir engineering, have also been examined (e.g., Talon et al. [30], Roustaei et al. [31], Bao et al. [32]). Combined inertial and non-Newtonian effects are adressed by Yan and Koplik [33]. All these works except [32] consider fractures with self-affine geometry.

The present work proceeds along the lines initiated by $[1,2]$ and investigates the fracture flow and conduction properties by solving the microscale Stokes and Laplace equations in three-dimensional fracture samples. Profit is taken of the tremendous increase of computational resources to widely extend the investigated range of parameters and to greatly improve the data accuracy, by using much larger fracture samples and statistical data sets, and by quantifying and correcting the discretization errors. The results of the solutions of the Stokes and the Laplace equations are systematically compared to those obtained with the Reynolds equations. Finally, based on the improved data set, heuristic expressions are 




(a)

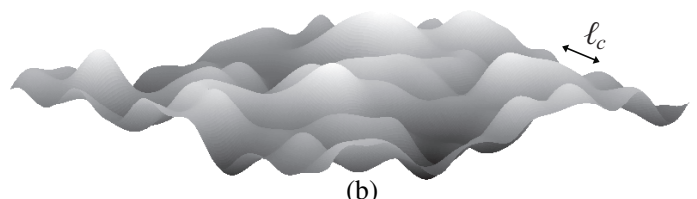

(b)

Fig. 1 (a) Convention and notations for the fracture geometry; the contact areas are displayed in black. (b) Three-dimensional view of a random surface with a Gaussian correlation.

proposed for the direct estimation of the transport coefficients as functions of the fracture geometrical characteristics, which could not be attempted in $[1,2]$.

\section{General}

\subsection{Description of fractures}

\subsubsection{Geometric statistical parameters}

A fracture is bounded by two non planar surfaces which may coincide over what is called a contact area. Such a fracture is schematized in Fig.1a. It is generally assumed that the two surfaces oscillate around two average planes $h_{0}^{ \pm}$, parallel to the $x y$-plane. The two surfaces $S_{p}^{+}$and $S_{p}^{-}$displayed in Fig.1a can be represented by the relations

$$
z^{ \pm}(\boldsymbol{x})=h_{0}^{ \pm}+h^{ \pm}(\boldsymbol{x})
$$

where $\boldsymbol{x}(x, y)$ is the position vector and $h^{ \pm}(\boldsymbol{x})$ the fluctuations of the surface around the average planes. The local aperture $b$ is the difference $w=z^{+}-z^{-}$when it is nonnegative

$$
b(\boldsymbol{x})=\left\{\begin{aligned}
w(\boldsymbol{x}), & w(\boldsymbol{x}) \geq 0 \\
0, & w(\boldsymbol{x})<0
\end{aligned}\right.
$$

When this aperture is equal to zero, it corresponds to the contact areas in Fig.1a.

The fluctuations $h^{ \pm}(\boldsymbol{x})$ are random in character. Let us first consider the average of these fluctuations. Two averages can be used, namely statistical and spatial averages which are denoted by brackets $\langle$.$\rangle and overbars \overline{(.)}$, respectively. For random fields which are 
statistically homogeneous, these two averages are equivalent. Since the statistical averages are more general, they are used in this Section.

The distance between the two average planes (for which $\left\langle h^{ \pm}(\boldsymbol{x})\right\rangle=0$ ) is denoted by

$$
b_{m}=h_{o}^{+}-h_{o}^{-}
$$

At least three other statistical characteristics are necessary to describe the random properties of a fracture. The first one is the probability densities $\varphi\left(h^{ \pm}\right)$of the fluctuations $h^{ \pm}(\boldsymbol{x})$. Experimental measurements generally show that these densities are Gaussian with identical roughness $\sigma_{h}[3,34-36]$

$$
\varphi\left(h^{ \pm}\right)=\frac{1}{\sqrt{2 \pi} \sigma_{h}} \exp \left[-\frac{h^{ \pm 2}}{2 \sigma_{h}^{2}}\right], \quad \sigma_{h}=\sigma_{h^{+}}=\sigma_{h^{-}}
$$

The second statistical characteristics is the spatial autocorrelation function of each surface

$$
C_{h^{ \pm}}(\boldsymbol{u})=\left\langle h^{ \pm}(\boldsymbol{x}) h^{ \pm}(\boldsymbol{x}+\boldsymbol{u})\right\rangle
$$

where $\boldsymbol{u}$ is the lag. The average is performed over $\boldsymbol{x}$. For isotropic surfaces, $C_{h^{ \pm}}$only depends on the norm $u$ of $\boldsymbol{u}$.

The third statistical characteristics is quantified by the intercorrelation coefficient $\theta_{I}$ between the two surfaces

$$
\theta_{I}=\frac{\left\langle h^{+}(\boldsymbol{x}) h^{-}(\boldsymbol{x})\right\rangle}{\sigma_{h}^{2}}
$$

From now on, the statistical properties of the upper and lower surfaces are identical and the superscript \pm is omitted.

\subsubsection{Autocorrelation function}

There are two major classes of autocorrelation functions $C_{h}(\boldsymbol{u})$, namely the Gaussian and the self-affine autocorrelations. The self-affine character of many rock surfaces was demonstrated by experimental observations (see e.g. [37]). Their transport properties have been investigated in $[13,14]$. Empirical but fairly accurate models for their conductivity and transmissivity have been formulated in the form of power laws of their fractional open area. We focus in this paper on the other classical case of Gaussian autocorrelations.

The Gaussian autocorrelation function for an isotropic fracture can be expressed as

$$
C_{h}(u)=\sigma_{h}^{2} \exp \left[-\left(\frac{u}{\ell_{c}}\right)^{2}\right]
$$

where $u$ is the norm of the lag $u$. $C_{h}(u)$ is characterized by a single correlation length scale $\ell_{c}$. The fracture surfaces with such an autocorrelation look like old mountains as in Fig.1b, and $\ell_{c}$ corresponds roughly to the distance between two summits or two valleys. The Gaussian surfaces are statistically homogeneous, or in other words they are statistically invariant by arbitrary translations.

Other surface rugosity indices than $\sigma_{h}$ are used in the literature, such as the RMS slope $Z_{2}$ along a profile [38]. For the Gaussian correlation (6), they are directly related by

$$
Z_{2}=\left\langle\left(\frac{\mathrm{d} h}{\mathrm{~d} x}\right)^{2}\right\rangle^{1 / 2}=2 \frac{\sigma_{h}^{2}}{l_{c}^{2}}
$$




\subsubsection{Fractional open area and mean aperture}

The fractional open area $S_{0}$ is the fraction of the total fracture surface where the aperture $b$ defined by (2) is strictly positive, or equivalently, it is the probability that $w$ is positive. The mean aperture $\bar{b}$ is the spatial average of $b$ over the whole fracture, including the contact zones. It is directly accessible to observation, for instance by measuring the fracture volume per unit area. These two quantities are related to the mean separation $b_{m}$ by [3]

$$
\begin{aligned}
S_{0} & =\frac{1}{2} \operatorname{erfc}\left(-\frac{b_{m}}{\sqrt{2} \sigma_{w}}\right) \\
\bar{b} & =S_{0} b_{m}+\frac{\sigma_{w}}{\sqrt{2 \pi}} \mathrm{e}^{-\frac{b_{m}^{2}}{2 \sigma_{w}^{2}}}
\end{aligned}
$$

where $\sigma_{w}$ is related to $\sigma_{h}$ by

$$
\sigma_{w}=\sigma_{h} \sqrt{2\left(1-\theta_{I}\right)}
$$

Note that these results apply regardless of the spatial correlation function $C_{h}$.

\subsubsection{Summary and generation}

If the surface roughness $\sigma_{h}$ is taken as the unit length, the dimensionless geometrical parameters which characterize the fracture are $b_{m} / \sigma_{h}, \ell_{c} / \sigma_{h}$, and $\theta_{I}$. Many experimental observations [35,39] are found in the intervals

$$
0.3 \lesssim \frac{\bar{b}}{\sigma_{h}} \lesssim 3, \quad 1 \lesssim \frac{\ell_{c}}{\sigma_{h}} \lesssim 7, \quad 0 \lesssim \theta_{I} \lesssim 1
$$

The generated fractures are spatially periodic in the $x$ - and $y$-directions, with a unit cell size $L \times L$, discretized into $N_{c} \times N_{c} \times N_{c z}$ elementary cubes of size $a$. The dimension $N_{c z}$ is set in order to encompass the whole open space $\tau_{0}$ of the fracture (see Fig.1a). Two uncorrelated random fields $h_{1}$ and $h_{2}$ are generated by the standard method of Fourier transforms (see [40, 41 ] for details), by imposing their variance $\sigma_{h}^{2}$ and the power spectrum of the covariance $C_{h}$, which depends on $l_{c}$. Then, fields $h^{+}$and $h^{-}$with the prescribed intercorrelation coefficient $\theta_{I}$ are obtained by a linear combination of $h_{1}$ and $h_{2}$. Note that the spatial correlations of the functions $h^{ \pm}$and of their difference $w$ are identical,

$$
\frac{C_{w}(u)}{\sigma_{w}^{2}}=\frac{C_{h}(u)}{\sigma_{h}^{2}}
$$

The two surfaces are then placed in the three-dimensional grid with their mean planes separated by the prescribed distance $b_{m}$. Finally, the discretized interstitial volume is obtained by retaining all the cubic volume elements whose centers rest between $S_{p}^{-}$and $S_{p}^{+}$.

It has to be mentioned that whereas this discretization procedure yields a correct fracture volume, i.e., a mean aperture $\bar{b}$ in agreement with (8), the fractional open area $S_{0}$ is slightly smaller than in the continuous space. The deviation can be quantified analytically and the complicated expression for the exact result is approximated with excellent precision by the simple statement that the fractional open area in the discrete model corresponds to $S_{0}$ as predicted by ( $8 \mathrm{a})$ for $\left(b_{m}-a / 2\right)$. The maximal deviation occurs for $b_{m}=0$, where $S_{0}$ is smaller than the theoretical value $1 / 2$ by $a /\left(2 \sqrt{2 \pi} \sigma_{w}\right)$, i.e., by 0.028 for $\theta_{I}=0$ and the discretization $a=\sigma_{h} / 5$ used in the simulations. 
In the following, the results for the fracture transport properties are analyzed in terms of the parameters $\sigma_{h}, l_{c}, \theta_{I}$ and $\bar{b}$, rather than $b_{m}$ since all these quantities can be measured in real fractures. The mean separation $b_{m}$ is used only in the discussion of technical issues such as discretization effects.

Examples of fracture samples with cell size $L=16 l_{c}$ and discretization $a=\sigma_{h} / 5$ are displayed in Fig.2, for various apertures, correlation lengths and intercorrelation coefficients.

\subsection{Transmissivity}

The low Reynolds number flow of an incompressible Newtonian fluid through a roughwalled channel is governed by the usual three-dimensional Stokes equations (see [1]) together with the no slip boundary condition at the solid surfaces

$$
\mu \nabla^{2} \mathbf{v}=\nabla p, \quad \nabla \cdot \mathbf{v}=0, \quad \mathbf{v}=0 \quad \text { on } S_{p}^{ \pm}
$$

where $\mathbf{v}, p$, and $\mu$ are the velocity, pressure, and viscosity of the fluid, respectively.

A macroscopic pressure gradient $\overline{\nabla p}$ is applied. Owing to the periodicity of the fracture geometry, the velocity field is periodic as well, with a period $L$ in the plane of the fracture.

When these equations are solved, the mean flow rate per unit fracture width $\boldsymbol{J}$ is derived and related to the mean pressure gradient by the $2 \times 2$ transmissivity tensor $\boldsymbol{\sigma}_{S}$

$$
\boldsymbol{J}=\frac{1}{L^{2}} \iiint_{\tau_{0}} \boldsymbol{v} \mathrm{d} x \mathrm{~d} y \mathrm{~d} z, \quad \boldsymbol{J}=-\frac{1}{\mu} \boldsymbol{\sigma}_{S} \cdot \overline{\nabla p}
$$

$\boldsymbol{\sigma}_{S}$ is homogeneous to the cube of a length and it only depends on the fracture geometry. The subscript $S$ indicates that $\boldsymbol{\sigma}_{S}$ is calculated by solving the Stokes equation. For isotropic fractures, $\boldsymbol{\sigma}_{S}$ is a spherical tensor $\sigma_{S} \boldsymbol{I}$ where $\boldsymbol{I}$ is the unit $2 \times 2$ tensor.

In reference to Poiseuille flow between parallel planes separated by a gap $b$, with transmissivity $b^{3} / 12$, the equivalent hydraulic aperture of a random channel can be defined by

$$
b_{S}=\left(12 \sigma_{S}\right)^{1 / 3}
$$

\subsection{Conductivity}

The conduction problem in the fracture is cast here in terms of diffusion, but the same formalism applies to electric current. In both cases, it is considered that the solid matrix is impervious or insulating, and that no surface diffusion or conduction takes place along the fracture boundaries.

The local concentration $c$ in the fracture void space $\tau_{o}$ is governed by the usual Laplace equation (see [2]) together with the no flux boundary condition

$$
\nabla^{2} c=0, \quad n \cdot \nabla c=0 \quad \text { on } S_{p}^{ \pm}
$$

where $\boldsymbol{n}$ is the normal vector to the solid surface $S_{p}^{ \pm}$. A macroscopic concentration gradient $\overline{\nabla c}$ is specified. Owing to the periodicity of the fracture geometry, the local concentration gradient is periodic as well, with a period $L$ in the plane of the fracture.

The mean flux per unit fracture width $\boldsymbol{J}_{c}$ can be derived by integrating the local flux over the volume $\tau_{o}$

$$
\boldsymbol{J}_{c}=-\frac{D}{L^{2}} \iiint_{\tau_{0}} \nabla c \mathrm{~d} x \mathrm{~d} y \mathrm{~d} z
$$




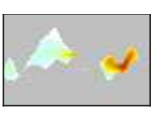

$l_{c} / \sigma_{h}=1$



$l_{c} / \sigma_{h}=2$

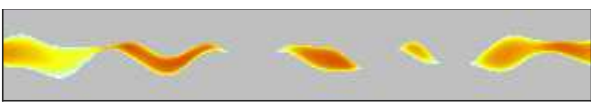

$l_{c} / \sigma_{h}=4$

$l_{c} / \sigma_{h}=8$
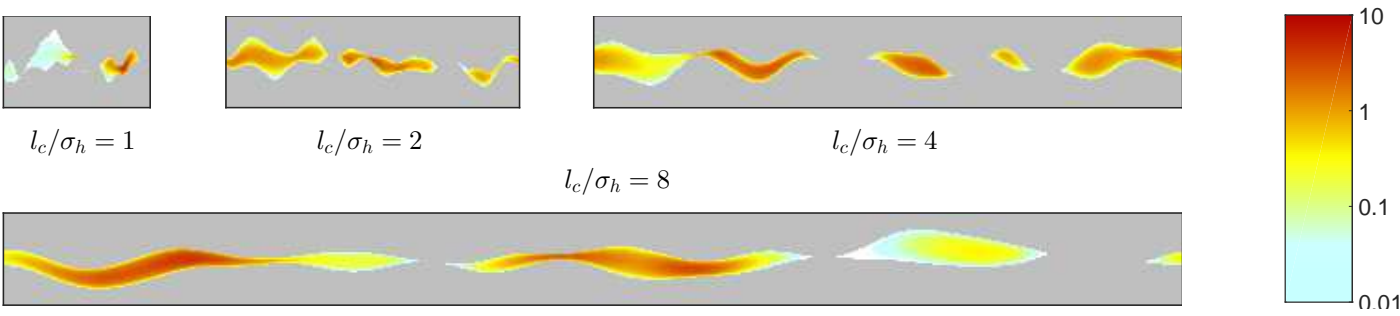

(a) $b_{m}^{\prime}=1, \theta_{I}=0$

(b)
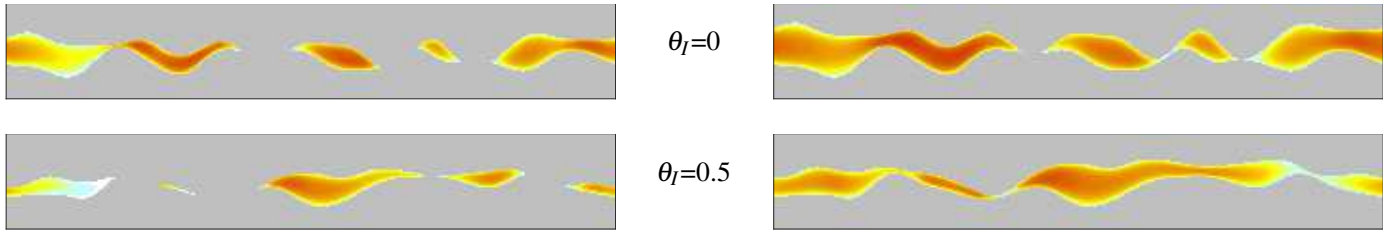

$\theta_{I}=0.5$
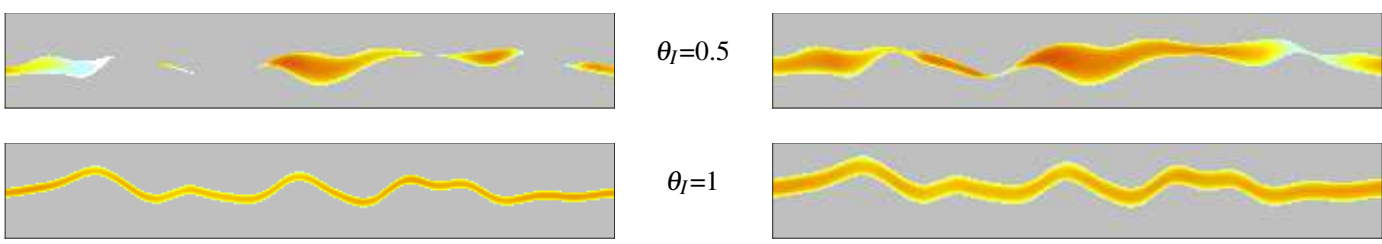

$\theta_{I}=1$

(c) $b_{m}^{\prime}=1, l_{c}^{\prime}=4$



(d) $b_{m}^{\prime}=2, l_{c}^{\prime}=4$

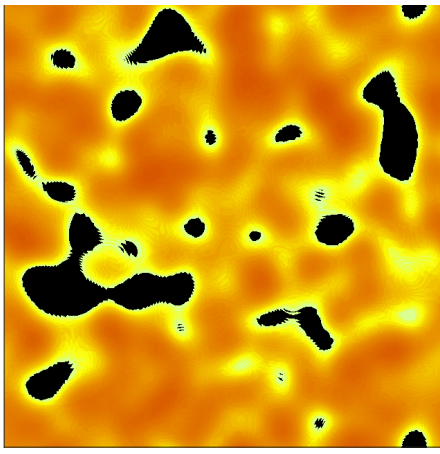

Aperture

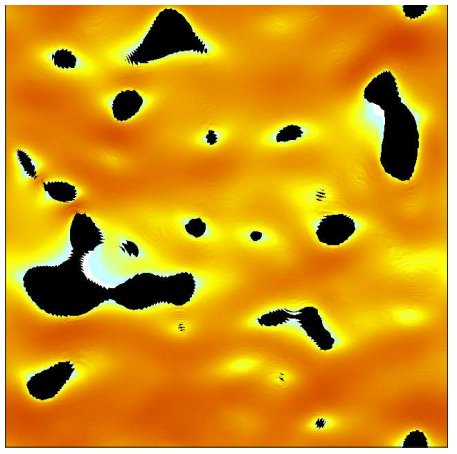

Diffusion flux



Flow rate

(e) $b_{m}^{\prime}=2, \theta_{I}=0, l_{c}^{\prime}=8$

Fig. 2 Cross-sections through fracture samples with $b_{m}^{\prime}=1, \theta_{I}=0$ and $l_{c}^{\prime}=1,2,4$ and 8 (a), and with $l_{c}^{\prime}=4, \theta_{I}=0$, 0.5 and 1 , and $b_{m}^{\prime}=1$ (c) or 2 (d). Top view of a fracture with $b_{m}^{\prime}=2, \theta_{I}=0$ and $l_{c}^{\prime}=8$ (e); the contact areas are shown in black. In all cases, $L^{\prime}=16$ and $a^{\prime}=0.2$. Colors correspond to the local flow velocity (a,c,d), and to the aperture, diffusive flux and flow rate integrated over the fracture aperture (e), with a driving gradient from left to right. All these quantities are normalized by their averages over the whole fracture and displayed with the logarithmic color code shown in (b). 
where $D$ is the molecular diffusion coefficient. Again, the mean flux is linearly related to the concentration gradient,

$$
\boldsymbol{J}_{c}=-\boldsymbol{\Lambda}_{L} \cdot \overline{\nabla c}
$$

For an isotropic fracture, the $2 \times 2$ conductivity tensor $\boldsymbol{\Lambda}_{L}$ becomes a spherical tensor $\Lambda_{L} \boldsymbol{I}$. The subscript $L$ indicates that $\boldsymbol{\Lambda}_{L}$ is deduced from the solution to the Laplace equation. $\Lambda_{L} / D$ is homogeneous to a length and it only depends on the geometry of the fracture.

In reference to the plane channel with aperture $b$ whose conductivity is equal to $D b$, an equivalent mean aperture for a random channel can be defined by

$$
b_{L}=\frac{\Lambda_{L}}{D}
$$

\subsection{Numerical procedure}

The partial differential equations (12) and (15) are discretized by finite differences in the $N_{c} \times N_{c} \times N_{c z}$ elementary cubes of size $a$ in which the fracture space is represented (cf Section 2.1.4).

As in [1], the so-called artificial compressibility method is used for the Stokes equations. The equations are solved successively along each direction by using an alternating direction implicit scheme. Convergence is reached when the flow rate is found to be the same within a precision of $10^{-3}$ across various sections of the medium. The Neuman problem (15) is solved via a second-order finite-difference formulation by using a conjugate-gradient method [2]. The conductivity of each random sample is determined with a precision of $10^{-6}$.

The calculations are repeated for a number $N_{r}$ of random realizations, with macroscopic gradients applied successively along the $x$ - and $y$-axes. The mean transport coefficients denoted by $\left\langle\sigma_{S}\right\rangle$ and $\left\langle\Lambda_{L}\right\rangle$ are deduced from the statistical averages of the macroscopic fluxes obtained in these $2 N_{r}$ calculations.

For Stokes flow, the typical values of $N_{r}$ range from 20 for large $b_{m}$ and $l_{c}$ (where the size of computational domain can reach $N_{c}=640$ and $N_{c z} \geq 100$ ) to 100 for smaller $l_{c}$ and intermediate $b_{m}$, and several hundreds for small apertures. Larger values were considered in some cases with large variability (up to 1600 when $b_{m}=\sigma_{h} / 2$ and $l_{c}=\sigma_{h}$ ), and sometimes smaller ones (but at least 10) when $l_{c} \gg \sigma_{h}$ with $\theta_{I} \geq 0.5$, where the statistical fluctuations are very small (see Fig.3). For the numerically less demanding Laplace problem, larger values of $N_{r}$ were applied, from at least 40 to several thousands for small $l_{c}$ or $b_{m}$.

\subsection{Notations}

The following notations are used in the presentation of the results.

The distinction between spatial averages denoted by an overbar $(e . g ., \bar{b})$ and statistical averages over a set of realizations denoted by brackets $\left(e . g .,\left\langle\sigma_{S}\right\rangle\right)$ should be kept in mind. Note that for the hydraulic aperture $b_{S}$, the improper but convenient notation $\left\langle b_{S}\right\rangle$ is used to designate $\left\langle 12 \sigma_{S}\right\rangle^{1 / 3}$, rather than $\left\langle\left(12 \sigma_{S}\right)^{1 / 3}\right\rangle$. Thus, $\left\langle b_{S}\right\rangle$ and $\left\langle\sigma_{S}\right\rangle$ are related by (14) and the results presented in terms of the mean hydraulic aperture can be straightforwardly transposed in terms of the mean transmissivity.

Dimensionless quantities denoted by primes are defined by normalization by geometric lengths associated with the surface characteristics, generally $\sigma_{h}$ except for the cell size $L$ for 

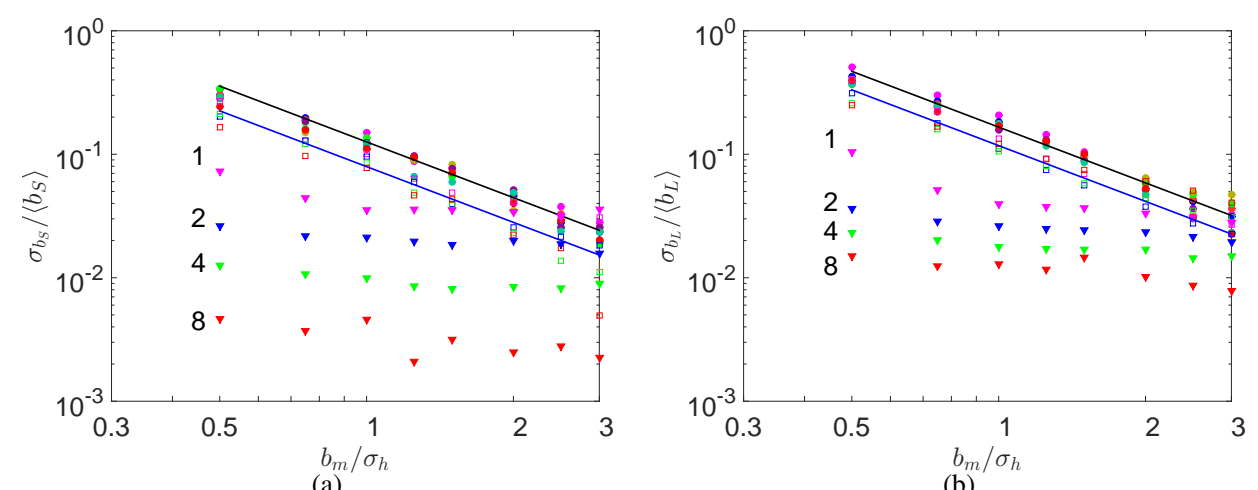

Fig. 3 The reduced standard deviations of the hydraulic aperture $\sigma_{b_{S}} /\left\langle b_{S}\right\rangle$ (a) and of the conductivity $\sigma_{b_{L}} /\left\langle b_{L}\right\rangle$ (b) as functions $b_{m} / \sigma_{h}$. Data are for $L=16 l_{c}, \theta_{I}=0(\bullet), 0.5(\mathbf{\square})$ and $1(\boldsymbol{\nabla})$, with $l_{c} / \sigma_{h}=1$ to 8 . The labels in the figures correspond to $l_{c} / \sigma_{h}$ for $\theta_{I}=1$. The thick solid lines correspond to Eq. (34) in Appendix A.

which $l_{c}$ is a more relevant scale,

$$
b_{m}^{\prime}=\frac{b_{m}}{\sigma_{h}}, \quad b^{\prime}=\frac{b}{\sigma_{h}}, \quad l_{c}^{\prime}=\frac{l_{c}}{\sigma_{h}}, \quad a^{\prime}=\frac{a}{\sigma_{h}}
$$

221

$$
L^{\prime}=\frac{L}{l_{c}}, \quad \sigma_{S}^{\prime}=\frac{\sigma_{S}}{\sigma_{h}^{3}}, \quad b_{S}^{\prime}=\frac{b_{S}}{\sigma_{h}}, \quad b_{L}^{\prime}=\frac{b_{L}}{\sigma_{h}}
$$

The transport coefficients are often compared to those of a plane channel with the same aperture $\bar{b}$. The corresponding dimensionless ratios are denoted by tildes,

$$
\widetilde{\sigma_{S}}=\frac{\sigma_{S}}{\bar{b}^{3} / 12}, \quad \widetilde{b_{S}}=\frac{b_{S}}{\bar{b}}, \quad \widetilde{b_{L}}=\frac{b_{L}}{\bar{b}}
$$

\section{Numerical results}

As discussed in Sections 2.2 and 2.3, the transport coefficients only depend on the geometry and one can write

$$
\sigma_{S}=f\left(\sigma_{h}, \bar{b}, l_{c}, \theta_{I}, a, L\right), \quad \Lambda_{L}=D g\left(\sigma_{h}, \bar{b}, l_{c}, \theta_{I}, a, L\right)
$$

This can be expressed in dimensionless form as

$$
\widetilde{\sigma_{S}}={\widetilde{b_{S}}}^{3}=f\left(\bar{b}^{\prime}, l_{c}^{\prime}, \theta_{I}, a^{\prime}, L^{\prime}\right), \quad \widetilde{b_{L}}=g\left(\bar{b}^{\prime}, l_{c}^{\prime}, \theta_{I}, a^{\prime}, L^{\prime}\right)
$$

There are two kinds of quantities among the arguments of $f$ and $g$ in (21). The first ones are real geometrical characteristics of the fracture, but the last two arguments are parameters without any physical relevance introduced in the numerical procedure. Their influence on the results should vanish if their are set appropriately, i.e., with a fine enough discretization step $a$ and a large enough unit cell size $L$. 
Unless otherwise stated, all calculations are conducted with a discretization step $a^{\prime}=0.2$, which was found satisfactory in earlier works $[1,2,13,14]$. The influence of $a$ on the numerical solution was carefully and thoroughly examined. The discretization error was quantified and a corrective procedure was devised and systematically applied to the results. The residual error in the predictions of $b_{S}$ and $b_{L}$ is estimated to be at most $\pm a / 10= \pm 0.02 \sigma_{h}$.

The cell size effects have also been quantified with great care. By comparison with data for larger sizes, the residual size effects associated with the value $L^{\prime}=16$ used in all systematic calculations are found to be in all cases of a much smaller amplitude than the statistical fluctuations.

Finally, the statistical fluctuations associated with the average results discussed in the following are presented in Appendix A. The standard deviations of $b_{S}$ and $b_{L}$ decay from a few tens of percent for small apertures to a few percent or less as the aperture increases, according to the power laws (34).

\subsection{Percolation}

Percolation of the interstitial space, i.e., the existence of a continuous path open to the flow across the whole fracture, is obviously a crucial parameter for its transport properties. This occurs when the fracture is sufficiently open, beyond a threshold which can be expressed in terms of $b_{m}, \bar{b}$ or $S_{0}$, in view of (8).

Percolation and the critical behavior of the fracture properties around the percolation threshold are out of the scope of the present study, but a short presentation of some basic results is useful for the discussion of the transport properties.

Two general features can be recalled. First, in a continuous description, percolation of the fracture interstitial volume is equivalent to the two-dimensional percolation of the projected open regions in the mean fracture plane. Second, the correlation length $\ell_{c}$ of Gaussian fractures only determines the scale of the contact and open zones, but not their topology; hence, $\ell_{c}$ does not influence the percolation characteristics. Thus, the percolation of the fracture open space depends only on $S_{0}$ or $b_{m} / \sigma_{w}$. The percolation of the projected open regions was carefully investigated in [41], with a fine resolution and elimination of the size effects by extrapolation to $L \rightarrow \infty$. The threshold was found very close to $S_{0 c}=1 / 2$, as expected in $2 \mathrm{~d}$ continuous percolation, which corresponds to $b_{m c}=0$ and $\bar{b}_{c}=\sigma_{w} / \sqrt{2 \pi}=\sqrt{\left(1-\theta_{I}\right) / \pi} \sigma_{h}$.

In this work, only fractures with $b_{m}^{\prime} \geq 1 / 2$ are considered, i.e., $S_{0} \geq 0.638$, significantly larger than $1 / 2$. However, a small fraction of the random realizations are found non percolating, for some combinations of the geometrical characteristics. This results from finite size effects, even though $L$ is relatively large, and from a discretization effect, since the percolation of the interstitial volume is not strictly equivalent to the percolation of the projected open areas in a discrete representation.

Non percolating samples occured only for the smallest investigated aperture $b_{m}^{\prime}=1 / 2$, for which up to $10 \%$ of the samples are found non percolating, and even $15 \%$ in the worst case $\left(\theta_{I}=0, b_{m}^{\prime}=1 / 2, l_{c}^{\prime}=1\right)$. These samples are included in the statistical sets for the averaging of the transport coefficients, with $\sigma_{S}=\Lambda_{L}=0$, whereas non zero values might be obtained with a smaller grid step. This may affect the statistical averages by a few percent, on top of the other discretization effects pertaining to the solution of the transport equations. 
3.2 Overview of the global trends

The global trends of the dependence of the transport coefficients on the geometrical parameters are illustrated in Fig.4. Data for $l_{c}^{\prime}=1$ to 8 are shown for uncorrelated surfaces with $\theta_{I}=0$ in $(\mathrm{a}, \mathrm{b})$ and for correlated surfaces with $\theta_{I}=0.5$ in $(\mathrm{c}, \mathrm{d})$ and $\theta_{I}=1$ in $(\mathrm{e}, \mathrm{f})$. Note that the standard deviations associated with these averages are given in Appendix A.

Of course, $\left\langle\widetilde{\sigma_{S}}\right\rangle$ and $\left\langle\widetilde{b_{L}}\right\rangle$ increase with the aperture in all cases and approach one when $\bar{b}^{\prime} \gg 1$, i.e., the fracture transport coefficients approach those of a plane channel. However, this convergence is slow, and $\left\langle\widetilde{\sigma_{S}}\right\rangle$ and $\left\langle\widetilde{b_{L}}\right\rangle$ are still significantly smaller than 1 when for instance $\bar{b}^{\prime}=6$, even though the fractures are virtually entirely open with a fractional contact area $1-S_{0}$ smaller than $10^{-5}$.

Both transport coefficients increase with $l_{c}^{\prime}$, i.e., when the aperture variations take place over longer distances (see Fig.2a). They also increase with $\theta_{I}$, i.e., as the fracture surfaces tend to be parallel. When both circumstances occur simultaneously, for instance with $\theta_{I}=1$ and $l_{c}^{\prime} \gg 1$ whatever the aperture, the transport coefficients are close to those of the plane channel with the same $\bar{b}$.

Note that the influence of the surface intercorrelation $\theta_{I}$ is strong for small apertures, but rapidly decreases as the aperture increases. This is confirmed by Fig.5, where $\left\langle\widetilde{\sigma_{S}}\right\rangle$ and $\left\langle\widetilde{b_{L}}\right\rangle$ are plotted as functions of $\theta_{I}$ for $l_{c}^{\prime}=4$ and various apertures. The transport coefficients are insensitive to the intercorrelation of the two surfaces when they are sufficiently far apart.

Examples of flow patterns are displayed in Fig.2. Excursions of the surfaces are seen in Fig.2a to create deep grooves which poorly contribute to the flow when $l_{c}$ is short. When $\theta_{I}$ increases (Fig.2c,d), the fractures become sinuous channels with parallel walls, where a nearly uniform flow can develop.

Some comparisons between the present data and those of $[1,2]$ are given in Fig.4a,b. The orders of magnitude are always found to be in good agreement. For instance, $\left\langle\widetilde{\sigma_{S}}\right\rangle$ of [1] almost coincides with the present values for $l_{c}^{\prime}=1$. However, there are discrepancies for $l_{c}^{\prime}=3$, which result primarily from statistical effects, due to the small number $N_{r}=5$ and size $L / l_{c}=3.33$ of the samples in [1], to the compared to $N_{r} \geq 100$ and $L / l_{c}=16$ for the present results.

\subsection{Relationship between the effective transport coefficients}

The effective apertures for flow and conduction are compared in Fig.6a,b where $\left\langle\widetilde{b}_{S}\right\rangle-\left\langle\widetilde{b}_{L}\right\rangle$ is plotted as a function of $\bar{b}^{\prime}$. The most salient feature is that this difference changes sign. Since $\left\langle\widetilde{b_{S}}\right\rangle-\left\langle\widetilde{b_{L}}\right\rangle$ increases with $l_{c}^{\prime}$ over the whole aperture range, the aperture where the change of sign takes place also increases, from $\bar{b}^{\prime} \approx 2$ for $l_{c}^{\prime}=1$ to $\bar{b}^{\prime} \approx 4$ for $l_{c}^{\prime}=8$.

At small apertures, $\left\langle\widetilde{b_{S}}\right\rangle$ is larger than $\left\langle\widetilde{b_{L}}\right\rangle$ due to the flow channelization. The fluid tends to flow along paths that sample the largest values of the aperture. This effect is much less significant for diffusion, as shown by the comparison of the flux maps in Figs.2d,f. The situation is reversed for large apertures, where channelization disappears. The surface roughness reduces the effective aperture open to the fluid flow in a greater respect than for diffusion, especially for small $l_{c}^{\prime}$ and therefore, $\left\langle\widetilde{b_{S}}\right\rangle<\left\langle\widetilde{b_{L}}\right\rangle$.

The intercorrelation coefficient $\theta_{I}$ strongly influences $\left\langle\tilde{b_{S}}\right\rangle-\left\langle\widetilde{b_{L}}\right\rangle$ at small aperture. The difference between the hydraulic and diffusion effective apertures decreases and eventually vanishes as $\theta_{I}$ increases and approaches 1 . However, it is remarkable that the influence of $\theta_{I}$ totally vanishes when $\bar{b}^{\prime} \gtrsim 4$ (Fig.6b). At large apertures, the disturbances due to the 


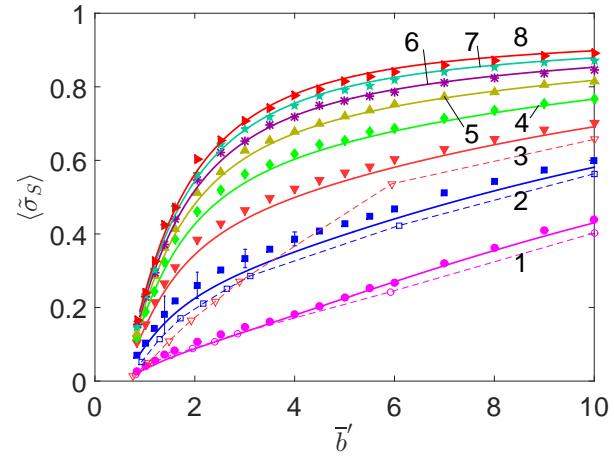

(a)

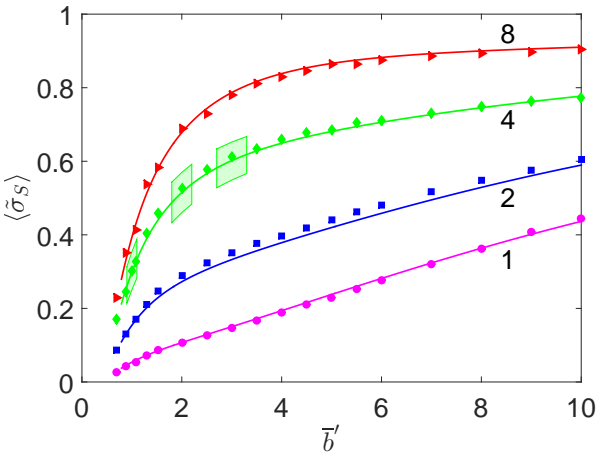

(c)

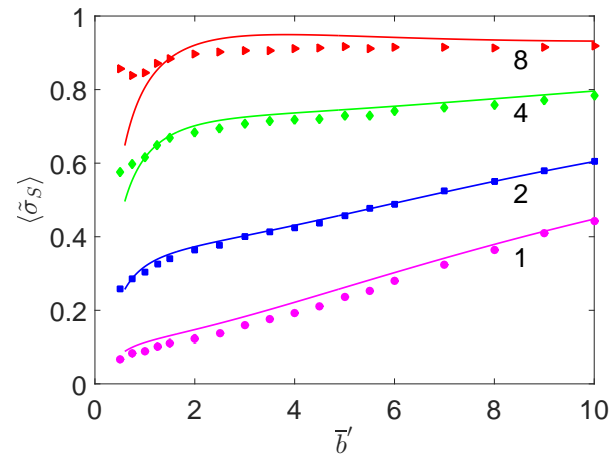

(e)

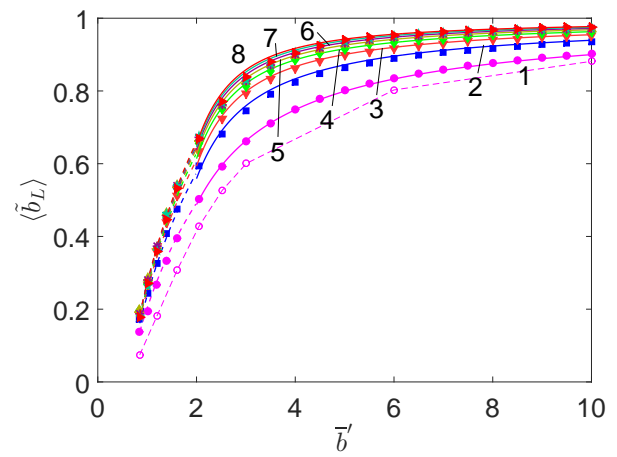

(b)

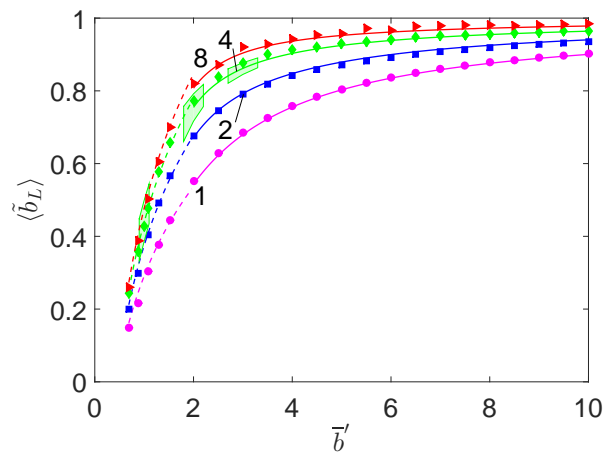

(d)

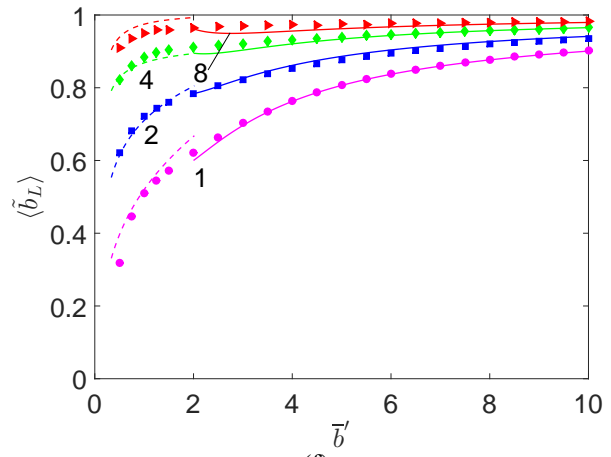

(f)

Fig. 4 The mean reduced transmissivity $\left\langle\widetilde{\sigma_{S}}\right\rangle(\mathrm{a}, \mathrm{c}, \mathrm{e})$ and conductivity $\left\langle\widetilde{b_{L}}\right\rangle(\mathrm{b}, \mathrm{d}, \mathrm{f})$ as functions of the aperture $\bar{b}^{\prime}$ for $\theta_{I}=0(\mathrm{a}, \mathrm{b}), 0.5(\mathrm{c}, \mathrm{d})$ and $1(\mathrm{e}, \mathrm{f})$. The labels correspond to $l_{c}^{\prime}$. The solid symbols correspond to the numerical data. The solid lines in (a,c,e) correspond to the model (32). The broken and solid lines in (b,d,f) correspond to the model (33). Open symbols joined by dash lines correspond to the data of [1] for $l_{c}^{\prime}=1,2$ and 3 in (a) and to the data of [2] for $l_{c}^{\prime}=1$ in (b). The boxes in (c,d) correspond to the uncertainty for $\left\langle{\widetilde{\sigma_{S}}}_{\rangle}\right\rangle$and $\left\langle\widetilde{b_{L}}\right\rangle$ if the geometrical characteristics are measured with a precision of $\pm 10 \%$ for $\bar{b}^{\prime}$ and $l_{c}^{\prime}$ and \pm 0.1 for $\theta_{I}$. 


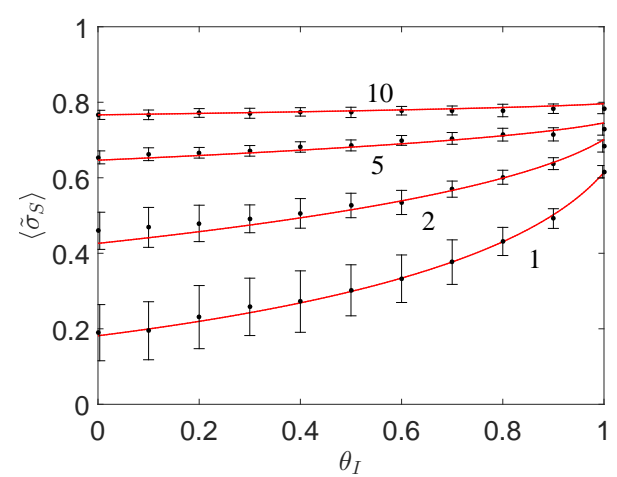

(a)

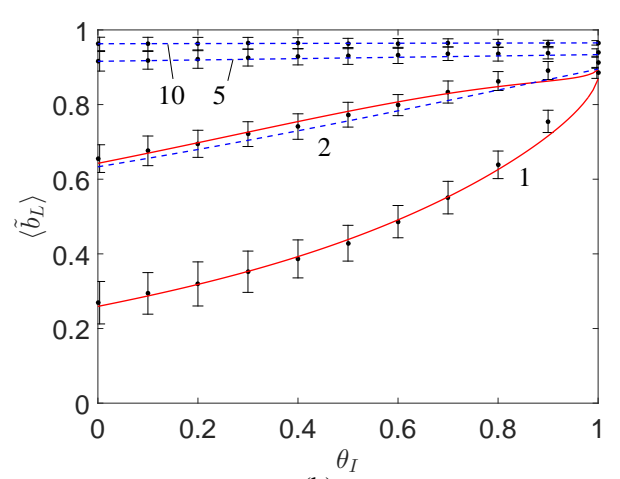

(b)

Fig. 5 The mean reduced transmissivity $\left\langle\widetilde{\sigma_{S}}\right\rangle$ (a) and conductivity $\left\langle\widetilde{b_{L}}\right\rangle$ (b) as functions of $\theta_{I}$ for $l_{c}^{\prime}=4$ and $\bar{b}^{\prime}=1,2,5$ and 10 . The symbols and error bars correspond to the mean numerical data and to \pm one standard deviation. The solid lines in (a) correspond to the model (32). The solid and broken lines in (b) correspond to the model (32) for $\bar{b}^{\prime} \leq 2$ and $\bar{b}^{\prime} \geq 2$, respectively. The labels in the figures correspond to $\bar{b}^{\prime}$.

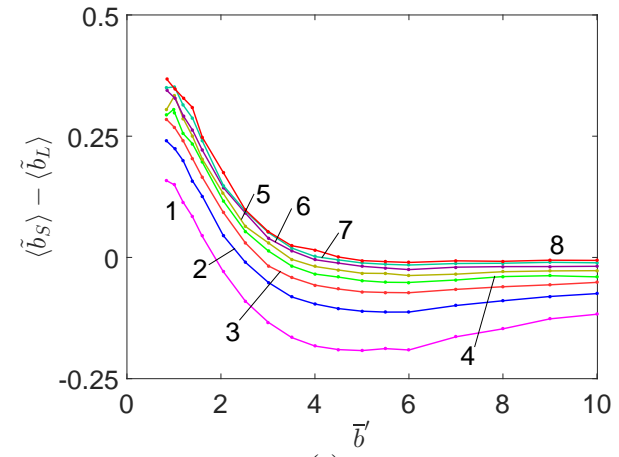

(a)

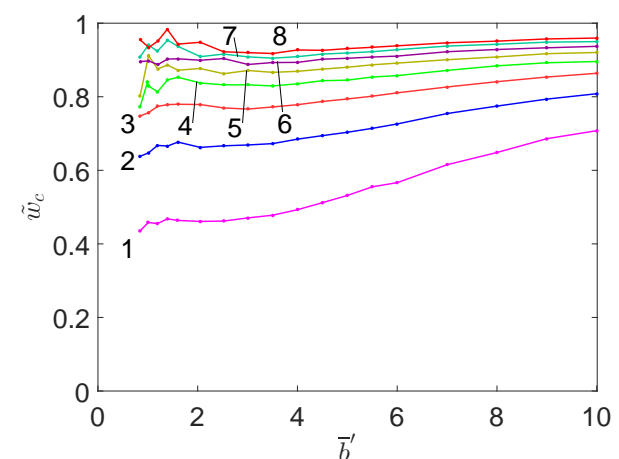

(c)

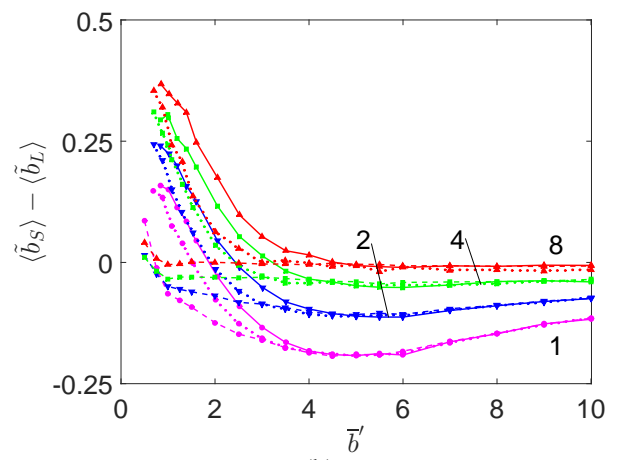

(b)

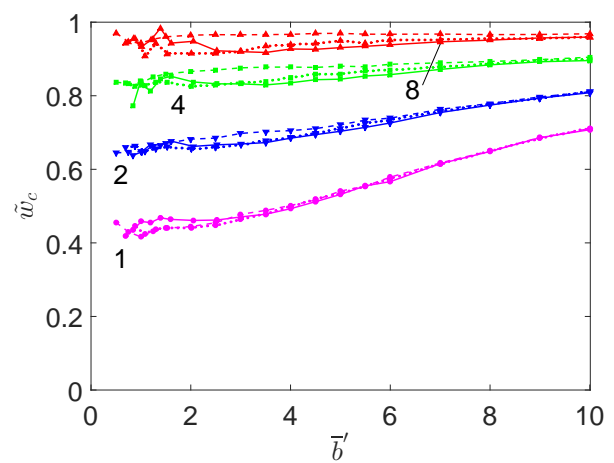

(d)

Fig. 6 The difference $\left\langle\widetilde{b_{S}}\right\rangle-\left\langle\widetilde{b_{L}}\right\rangle(\mathrm{a}, \mathrm{b})$ and the length $\widetilde{w_{c}}(\mathrm{c}, \mathrm{d})$ as functions of $\bar{b}^{\prime}$. Data in (a,c) are for $\theta_{I}=0$ and data in (b,d) are for $\theta_{I}=0$ (solid lines), 0.5 (dotted lines) and 1 (dash lines). The labels in the figures correspond to $l_{c}^{\prime}$. 
roughness of the two fracture surfaces do not interact, and they both result in an apparent shift of the mean surface planes. This limit is examined in detail in Section. 3.5.1.

Another comparison can be made by considering the ratio of the flow and diffusion transport coefficients. In three-dimensional porous media, the ratio of the permeability $K$ to the effective diffusivity $\bar{D}$ is often successfuly predicted by a semi-empirical model based on a percolation theory argument [42]

$$
\frac{K}{\bar{D}}=\mathscr{C} w_{c}^{2}
$$

and the mass conservation equation yields

$$
\nabla \cdot\left(b^{3} \nabla p\right)=0
$$

347 For diffusion, the local flux $\boldsymbol{j}_{c}$ is given by

$$
\boldsymbol{j}_{c}=-b D \nabla c
$$


and if the molecular diffusion coefficient is constant, the Reynolds equation reads

$$
\nabla \cdot(b \nabla c)=0
$$

These equations should be supplemented with overall boundary conditions, such as prescribed macroscopic pressure or concentration gradient, $\overline{\nabla p}$ or $\overline{\nabla c}$, together with spatial periodicity, as in the 3D Stokes or Laplace problems.

After solution of the 2D problems (25) or (26) over the $L \times L$ domain, integration of the two dimensional fluxes over the fracture surface yields the transmissivity $\sigma_{R}$ and the conductivity $\Lambda_{R}$ where the subscript $R$ refers to the Reynolds approximation.

The Reynolds approximations for flow and conductivity are compared to the Stokes and Laplace solutions in Fig.7 for $\theta_{I}=0,0.5$ and 1. Note that no systematic correction of discretization errors was applied for the solutions of Reynolds equation. However, it was checked by successive refinements in some cases that these errors are generally negligible and reach at most $3 \%$ for $\sigma_{R}$ for very small apertures with $\theta_{I}=0$.

When the correlation length $\ell_{c}^{\prime}$ and the aperture $\bar{b}^{\prime}$ are large, the Reynolds approximation is valid and the ratios approach 1 . However, realistic values of these parameters for real fractures are in the range given by (10) and indicated by frames in Figs.7a,b where large deviations between the two results are visible.

The maximal error is observed for small aperture $\bar{b}^{\prime} \approx 1$ and short correlation length $\ell_{c}^{\prime}=1$. The ratio $\left\langle\sigma_{R}\right\rangle /\left\langle\sigma_{S}\right\rangle$ is about 6.6 and $\left\langle\Lambda_{R}\right\rangle /\left\langle\Lambda_{S}\right\rangle$ is about 1.4 when $\theta_{I}=0$. Reynolds approximation widely overestimates both transport coefficients. Interestingly, $\left\langle\sigma_{R}\right\rangle /\left\langle\sigma_{S}\right\rangle$ is still larger for mutually correlated surfaces, with $\left\langle\sigma_{R}\right\rangle /\left\langle\sigma_{S}\right\rangle \approx 9.4$ and $\left\langle\Lambda_{R}\right\rangle /\left\langle\Lambda_{S}\right\rangle \approx 1.8$ when $\theta_{I}=0.5$ and $\left\langle\sigma_{R}\right\rangle /\left\langle\sigma_{S}\right\rangle \approx 12$ and $\left\langle\Lambda_{R}\right\rangle /\left\langle\Lambda_{S}\right\rangle \approx 2.2$ when $\theta_{I}=1$.

The influence of $\theta_{I}$ vanishes for large apertures and/or correlation length, roughly speaking when $\bar{b}^{\prime} l_{c}^{\prime} \gtrsim 8$. However, large deviations remain even in very open channels if the roughness correlation length is short, with $\left\langle\sigma_{R}\right\rangle /\left\langle\sigma_{S}\right\rangle \approx 2.7$ and $\left\langle\Lambda_{R}\right\rangle /\left\langle\Lambda_{S}\right\rangle \approx 1.13$ for all $\theta_{I}$ when $\bar{b}^{\prime}=8$ and $\ell_{c}^{\prime}=1$. A fair agreement is reached only for a slowly varying aperture which is the a priori condition of validity of the lubrication approximation. When $l_{c}^{\prime}=8$, the solution of Reynolds equation overestimates $\left\langle\sigma_{S}\right\rangle$ by about $10 \%$ and $\left\langle\Lambda_{S}\right\rangle$ by $2 \sim 3 \%$, for all $\bar{b}^{\prime}$ and $\theta_{I}$.

\subsection{Modelization of the transport coefficients}

Models are proposed in this Section to represent the numerical data, and provide a convenient mean for the direct estimation of the fracture transport coefficients as functions of their geometrical parameters. Expressions are given first in the limiting cases of large and small apertures. Then, models are devised which represent the data with a fairly good accuracy over the whole range of the parameters. It should be noted that the forms of the expressions are heuristic, and justified mostly by their practical success.

\subsubsection{Large apertures}

In the limit of large apertures, the fractures can be viewed as plane channels with rough walls, and the data can be analyzed in terms of the apparent reductions $\delta b_{S}$ and $\delta b_{L}$ of their aperture due to the surface roughness,

$$
\delta b_{S}=\bar{b}-b_{S}, \quad \delta b_{L}=\bar{b}-b_{L}
$$




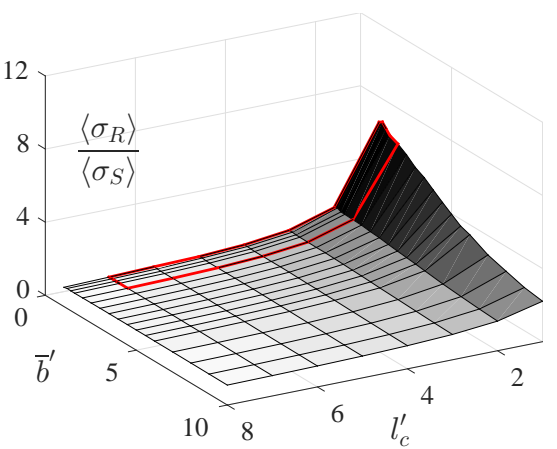

(a)

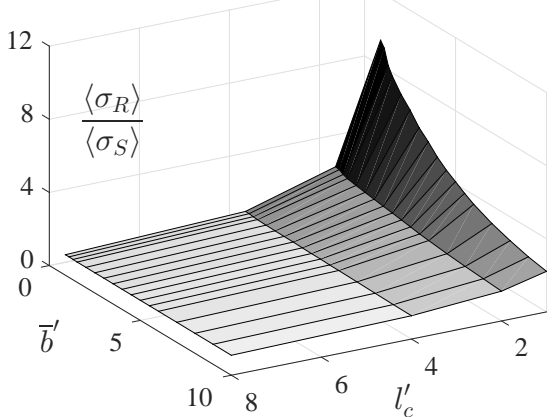

(c)

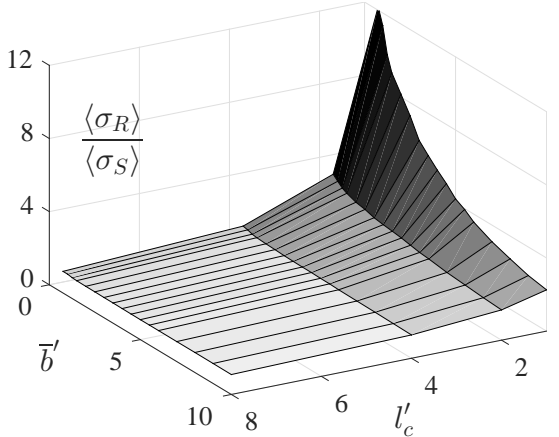

(e)

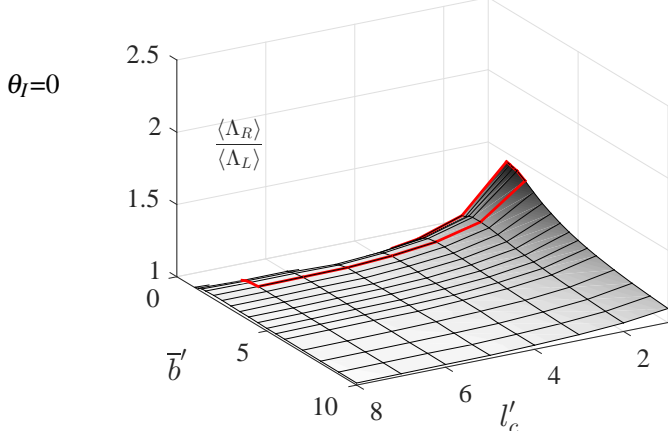

(b)

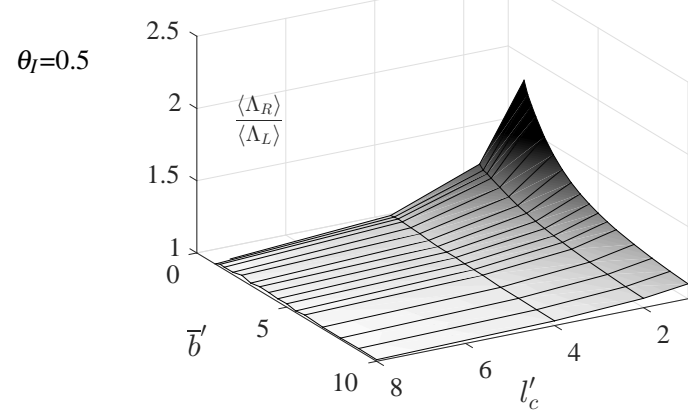

(d)



Fig. 7 Comparisons of the transport coefficients resulting from the Stokes (left column) or Laplace (right column) equations with the predictions of Reynolds equation. Data are for: $\theta_{I}=0$ (a, b), 0.5 (c, d) and 1 (e, f). Red thick lines in $(a, b)$ indicate the ranges (10) of commonly observed values of $\bar{b}^{\prime}$ and $\ell_{c}^{\prime}$.

These quantities are plotted in Fig.8. They converge toward a limit when $\bar{b}$ increases. This limit is a decreasing function of the correlation length $l_{c}^{\prime}$ but does not depend on the intercorrelation coefficient $\theta_{I}$; as suggested by Fig.8c, it can be modeled by the power laws

$$
\delta b_{S}^{\prime} \approx 3 l_{c}^{\prime-3 / 4}, \quad \delta b_{L}^{\prime} \approx l_{c}^{\prime-3 / 4} \quad\left(\bar{b}^{\prime} \rightarrow \infty\right)
$$

The influence of the surface roughness on the transmissivity is about three times larger than on conductivity. 




(a)

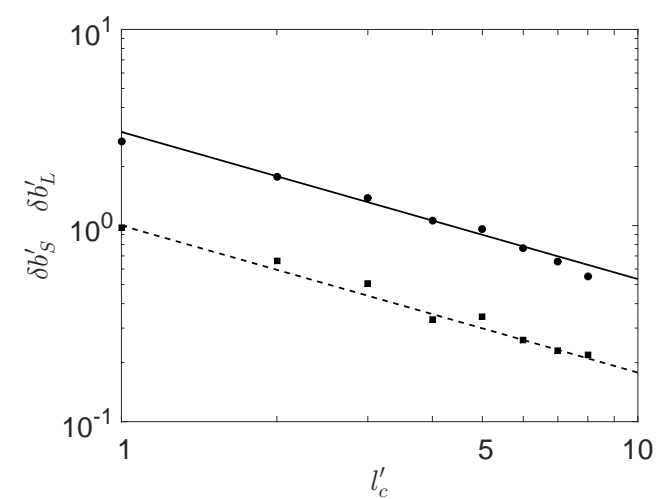

(c)

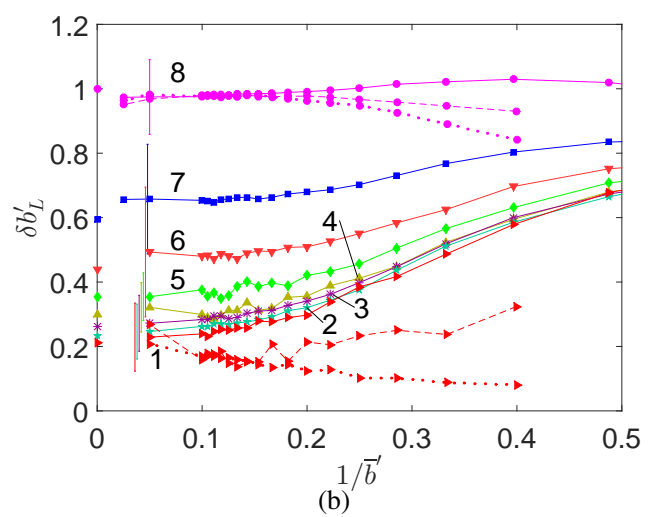

(b)

Fig. 8 The reductions $\delta b_{S}^{\prime}$ (a) and $\delta b_{L}^{\prime}$ (b) of the effective aperture for the transport processes at large apertures as functions of $1 / \bar{b}^{\prime}$, for $\theta_{I}=0$ and $l_{c}^{\prime}=1$ to 8 (solid lines). Data are also shown for $\theta_{I}=0.5$ (dotted lines)

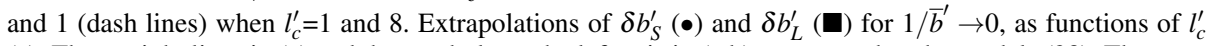
(c). The straight lines in (c) and the symbols on the left axis in (a,b) correspond to the models (28). The error bars shown in some cases correspond to \pm one standard deviation. The labels in the figures correspond to $l_{c}^{\prime}$.

\subsubsection{Small apertures}

Recall that the behavior of the fracture transport properties at the percolation threshold is not specifically addressed in this study. We consider here apertures in the range $0.5 \lesssim \bar{b}^{\prime} \lesssim 2$.

The numerical data for the transmissivity $\left\langle\sigma_{S}^{\prime}\right\rangle$ are shown in logarithmic scales in Fig.9 (left column), as functions of the aperture $\bar{b}^{\prime}$ shifted by an offset value. For all values of $\theta_{I}$, the data appear to obey cubic laws, which can be summarized by

$$
\left\langle\sigma_{S}^{\prime}\right\rangle \approx \frac{1}{0.8\left(1+7 / l_{c}^{\prime 2}\right)} \frac{\left[\bar{b}^{\prime}-0.4 \sqrt{1-0.94 \theta_{I}}\right]^{3}}{12}
$$

This model involves a prefactor that depends on the correlation length, and an offset for the aperture that depends on the intercorrelation coefficient $\theta_{I}$. This offset does not exactly 
correspond to the theoretical percolation threshold which is $\bar{b}_{c}^{\prime}=\sqrt{\left(1-\theta_{I}\right) / \pi}$ (for $S_{0}=$ $1 / 2$ ), but does not strongly deviate from it and depends on $\theta_{I}$ in a similar way.

Similar plots for the conductivity are shown in the right column of Fig.9. They reveal a more complex behavior. Power laws are still observed, but their exponent decreases from 1.35 when $\theta_{I}=0$ to 1 when $\theta_{I}=1$ with short $l_{c}^{\prime}$. The prefactor also depends on both $l_{c}^{\prime}$ and $\theta_{I}$. Combining fitted models for the exponent, aperture offset and prefactor results in

$$
\begin{aligned}
& \left\langle b_{L}^{\prime}\right\rangle \approx \frac{0.84+0.31 \theta_{I}}{1+\left(0.41+0.7 \theta_{I}\right) / l_{c}^{\prime}} \\
& \quad \times\left[\bar{b}^{\prime}-0.55 \sqrt{1-0.996 \theta_{I}}\right]^{1+0.35\left(1-\frac{\theta_{I}^{3}}{1+12 / l_{c}^{\prime 4}}\right)}
\end{aligned}
$$

The offset for the aperture slightly differs from that in (29) and is found very close to $\bar{b}_{c}^{\prime}$.

Models (29) and (30) are plotted in Fig.9 in comparison with the numerical data.

\subsubsection{Overall Models}

There is no obvious way to model the transport coefficients for intermediate apertures between the two limiting cases examined in the above. Besides, different situations are observed for the transmissivity, where cubic laws are always observed in both limiting cases and for the conductivity, where the exponent varies with $l_{c}^{\prime}$ and $\theta_{I}$. Hence, different heuristic approaches have been applied in each case.

Note first that the existence of cubic laws with different prefactors for the transmissivity at small and large apertures separated by a transition regime in the intermediate range has already been observed by [43], who considered fractures with self-affine correlations of the apertures and used the Reynolds approximation to evaluate the effective transmissivity. However, there are differences. First, the transmissivity at small apertures, is found in [43] to vary as the cube of the aperture of the tightest constriction that the flowing fluid has to cross whereas the cubic law (29) is in terms of the mean aperture $\bar{b}^{\prime}$, and there is no straightforward way to relate the former to the latter. Secondly, the scaling properties of the aperture field in [43] allows a different but well defined power law regime to prevail over a wide range of intermediate apertures, whereas the transition takes place over a shorter range without obvious law in the present situation.

It was therefore chosen to represent the variations of $\left\langle\widetilde{\sigma_{S}}\right\rangle$ over the whole range of apertures by combining (28) and (29) with a weighting function $\psi$ that ranges from 0 (large apertures) to 1 (small apertures)

$$
\psi=\frac{1}{1+\left(\bar{b}^{\prime} / 8\right)^{2}}
$$

A cubic law is built with a prefactor and an offset which are both deduced from those for the small and large aperture limits, by applying coefficients $\psi$ and $(1-\psi)$ to the parameters of (29) and (28), respectively. Note that (28) is also replaced by the modified expression $\delta b_{S}^{\prime} \approx$ $2 l_{c}^{\prime-0.6}$, which is more representative of the observations in the upper range of apertures for practical applications than the limit (28) for $\bar{b}^{\prime} \rightarrow \infty$. The resulting model reads

$$
\begin{aligned}
& \left\langle\widetilde{\sigma_{S}}\right\rangle=\left[\frac{\psi}{0.8\left(1+7 / l_{c}^{\prime 2}\right)}+(1-\psi)\right] \\
& \times\left[1-\left\{\psi \frac{0.4 \sqrt{1-0.94 \theta_{I}}}{\bar{b}^{\prime}}+(1-\psi) \frac{2 l_{c}^{\prime-0.6}}{\bar{b}^{\prime}}\right\}\right]^{3}
\end{aligned}
$$




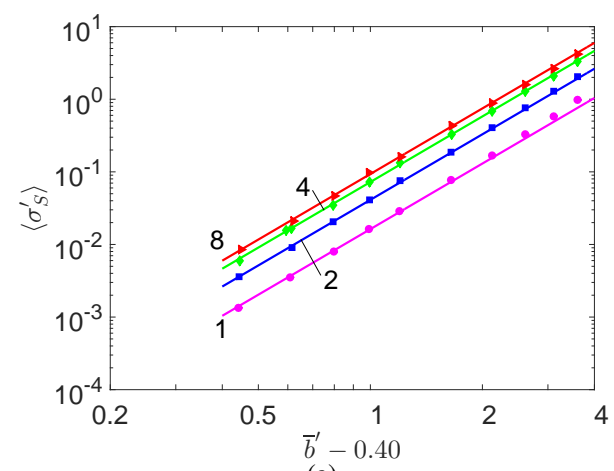

(a)

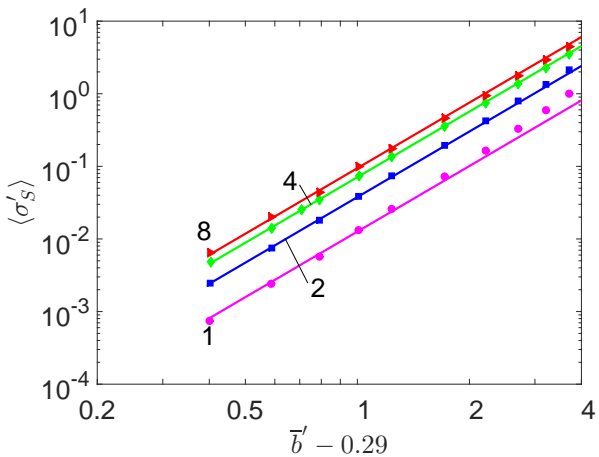

(c)

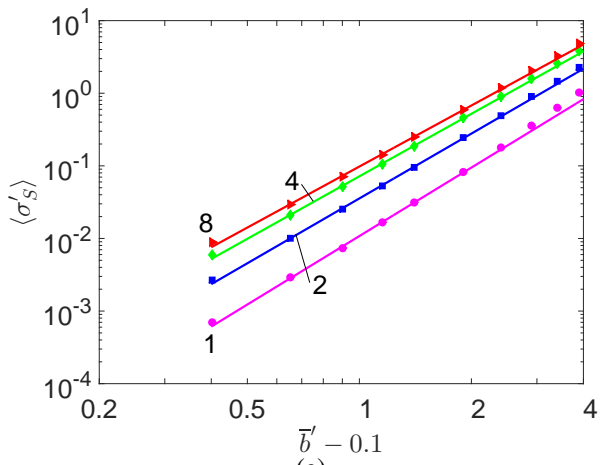

(e)

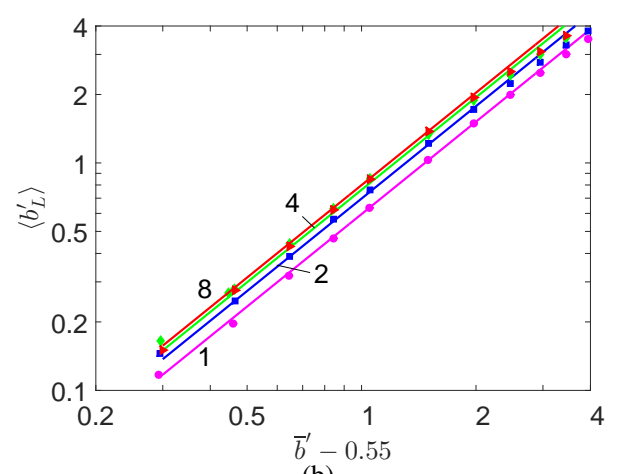

(b)



(d)

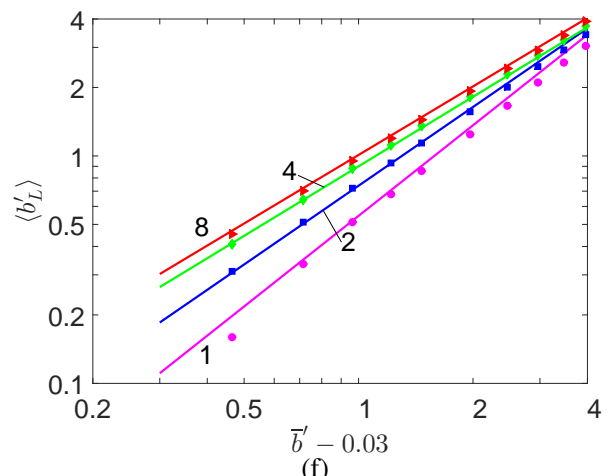

Fig. 9 The mean reduced transport coefficients $\left\langle\sigma_{S}^{\prime}\right\rangle$ (a,c,e) and $\left\langle b_{L}^{\prime}\right\rangle$ (b,d,f) as functions of the aperture $\bar{b}^{\prime}$ shifted by an offset, for $\theta_{I}=0(\mathrm{a}, \mathrm{b}), 0.5(\mathrm{c}, \mathrm{d})$ and $1(\mathrm{e}, \mathrm{f})$. The symbols correspond to the numerical data and the solid lines correspond to the models $(29,30)$. The labels in the figures correspond to $l_{c}^{\prime}$. 
The comparison in Figs. 4 and 5 of (32) with the numerical data is gratifying. The trends of the influences of all the geometrical parameters is captured, and the quantitative agreement is satisfactory. The relative deviations are generally of a few percents; they reach $10 \sim 20 \%$ only for small apertures when $l_{c}^{\prime}=\theta_{I}=1$ and they always remain within the range of \pm one standard deviation of the statistical fluctuations. They are also smaller than the uncertainties associated with the determination of the geometrical parameters in practical applications, which are illustrated in Fig.4c for a few typical cases in the range of (10) if $\bar{b}^{\prime}$ and $l_{c}^{\prime}$ are measured within $\pm 10 \%$ and $\theta_{I}$ within \pm 0.1 . Thus, (32) is a convenient model for a direct and easy evaluation of the fracture transmissivity.

Because of the more complex form of (30) compared to (29), a different approach is applied to conductivity. Figure 9 shows that (30) applies with reasonable accuracy up to apertures $\bar{b}^{\prime} \lesssim 2$. Conversely, an expression for $b_{L}$ applicable in the range $\bar{b}^{\prime} \gtrsim 2$ can be devised by supplementing the corrective term $\delta b_{L}$ in (28) with a higher order correction. This results in the model

$$
\begin{aligned}
& \left\langle\widetilde{b_{L}}\right\rangle=\frac{0.84+0.31 \theta_{I}}{1+\frac{0.41+0.7 \theta_{I}}{l_{c}^{\prime}} \bar{b}^{0.35}\left(1-\frac{\theta_{I}^{3}}{1+\frac{12}{l_{c}^{\prime 4}}}\right)} \\
& \times\left[1-\frac{0.55 \sqrt{1-0.996 \theta_{I}}}{\bar{b}^{\prime}}\right]^{1+0.35\left(1-\frac{\theta_{I}^{3}}{1+\frac{12}{l_{c}^{4}}}\right)} \quad\left(\bar{b}^{\prime} \lesssim 2\right) \\
& \left\langle\widetilde{b_{L}}\right\rangle=1-\frac{l_{c}^{\prime-3 / 4}}{\bar{b}^{\prime}} \\
& -\left[\left(2.2-2.7 \theta_{I}\right)-\frac{2.1-1.8 \theta_{I}}{l_{c}^{\prime}}\right] \frac{1}{\bar{b}^{\prime 3}} \quad\left(\bar{b}^{\prime} \gtrsim 2\right)
\end{aligned}
$$

Again, the comparisons in Figs.4 and 5 of (33) with the numerical data show a good

agreement. The relative deviations are smaller than $\pm 3 \%$ for $\bar{b}^{\prime} \geq 2$, smaller than $\pm 5 \%$ for $\bar{b}^{\prime}<2$ except for a few outliners at very small apertures, and within \pm one standard deviation of the statistical fluctuations. They are also smaller than the uncertainties associated with the determination of the geometrical parameters, illustrated in Fig.4c for few typical cases.

\section{Concluding remarks}

The effective flow and conduction properties of fractures with Gaussian spatial correlations have been investigated by solving the microscale governing equations in three-dimensional samples, along the lines initiated by $[1,2]$ but in greater details, over a wider range of the parameters, and with greatly improved accuracy. The effective transport coefficients have been related to the geometrical characteristics, quantified by the mean aperture (fracture volume per unit projected area), the surface roughness RMS amplitude, its correlation length, and the intercorrelation coefficient of the roughness on the two surfaces.

Since these parameters are intrinsic, model-independent characteristics of the fracture geometry, the numerical data and the various modelizations which have been proposed are probably applicable with reasonable accuracy to fractures that do not strictly comply with 
the Gaussian model. A check of this conjecture and if necessary a generalization of the predictive models to account for other types of short-range spatial correlations are a natural and straightforward extension of the present study.

Acknowledgements Part of this work was performed when P.M.A. was supported at the Mechanical Engineering Department, Technion, Haifa, Israel, by a fellowship of the Lady Davis Foundation.

\section{Appendix A Statistical fluctuations}

The statistical fluctuations of the transport coefficients are summarized in Fig.3 for all the calcultations with $L=16 l_{c}$. The reduced standard deviations of the hydraulic aperture $\sigma_{b_{S}} /\left\langle b_{S}\right\rangle$ and of the conductivity $\sigma_{b_{L}} /\left\langle b_{L}\right\rangle$ are plotted as functions $b_{m} / \sigma_{h}$ for $l_{c} / \sigma_{h}=1$ to 8 with $\theta_{I}=0,0.5$ and 1 .

When $\theta_{I}=0$ and 0.5 , the relative fluctuations are decreasing functions of the aperture for both transport processes, with only a very small influence of the correlation length $l_{c}$. They roughly follow the power laws

$$
\frac{\sigma_{b_{S}}}{\left\langle b_{S}\right\rangle} \approx 0.13\left[\frac{b_{m}}{\sigma_{h}}\right]^{-\frac{3}{2}}, \quad \frac{\sigma_{b_{L}}}{\left\langle b_{L}\right\rangle} \approx 0.17\left[\frac{b_{m}}{\sigma_{h}}\right]^{-\frac{3}{2}}
$$

for uncorrelated surfaces $\left(\theta_{I}=0\right)$ and

$$
\frac{\sigma_{b_{S}}}{\left\langle b_{S}\right\rangle} \approx 0.08\left[\frac{b_{m}}{\sigma_{h}}\right]^{-\frac{3}{2}}, \quad \frac{\sigma_{b_{L}}}{\left\langle b_{L}\right\rangle} \approx 0.12\left[\frac{b_{m}}{\sigma_{h}}\right]^{-\frac{3}{2}}
$$

for correlated surfaces with $\theta_{I}=0.5$.

A different behaviour prevails for $\theta_{I}=1$. The fluctuations decrease only slightly with the aperture, and much more significantly with the correlation length. However, the magnitude of the fluctuations is smaller than when $\theta_{I}<1$. They are generally a few percents or less, except for very small apertures and short correlation lengths, and never exceed $10 \%$.

\section{References}

1. Mourzenko, V.V., Thovert, J.-F. and Adler, P.M., J. Phys. II 5, 465-482 (1995)

2. Volik S., V.V. Mourzenko, J.-F. Thovert and P.M. Adler, Trasnp. in Porous Media 27, 305-325 (1997)

3. Adler P.M. and J.-F. Thovert, Fractures and Fracture Networks (Kluwer Academic Publishers, Dordrecht, Netherlands, 1999)

4. Zimmerman, R.W. and I. Main, in Gueguen, Y. and Bouteca, M. (eds.), Mechanics of fluid-saturated rocks, Elsevier Academic Press, London, 2004) 363-422

5. Patir N. and H.S. Cheng, J. Lubr. Technol. 100, 12-17 (1978)

6. Zimmerman R.W., S. Kumar and G.S. Bodvarson, Int. J. of Rock Mech. and Min. Sci. and Geomech. Abstr. 28, 325-331 (1991)

7. Witherspoon P.A., J.S.Y. Wang, K. Iwai and J.E. Gale, Water Resours. Res. 16, 1016-1024 (1980)

8. Tsang Y.W. and P.A. Witherspoon, J. Geophys. Res. B86, 9287-9298 (1981)

9. Gutfraind R. and A. Hansen, Transp. Porous Media 18, 131-149 (1995)

10. Brown S.R., H.W. Stockman and S.J. Reeves, Geophys. Res. Lett. 22, 2537-2540 (1995)

11. van Genabeek, O. and D.H. Rothman, Physics Letters A 255, 31-36 (1999)

12. Madadi, M. and M. Sahimi, Phys. Rev. E 67, 026309 (2003)

13. Mourzenko, V.V., J.-F. Thovert and P.M. Adler, Phys. Rev. E E59, 4265-4284 (1999)

14. Mourzenko, V.V., J.-F. Thovert and P.M. Adler, Transp. Porous Media 45, 89-103 (2001)

15. Oron, A. P. and B. Berkowitz, Water Resources Research 34, 2811-2825 (1998)

16. Drazer, G. and J. Koplik, Physical Review E 62, 8076-8085 (2000) 
17. Koyama, T., I. Neretnieks and L. Jing, International Journal of Rock Mechanics, Mining Sciences \& Geomechanical Abstracts 45, 1082-1101 (2008)

18. Basha, H. A. and W. El-Asmar, Water Resources Research 39, 1365, doi:10.1029/2003WR002472 (2003)

19. Crandall, D., G. Ahmadi and D.H. Smith, Transport in Porous Media 84, 493-510 (2010)

20. Sisavath, S., A. Al-Yaarubi, C.C. Pain and R.W. Zimmerman, Pure and Applied Geophysics 160, 1009$1022(2003)$

21. Malevich A.E., V. Mityushev and P.M. Adler, Acta Mechanica 182, 151 (2006)

22. Adler P.M., A.E. Malevich and V.V. Mityushev, Acta Mech. 224, 1823 (2013)

23. Plouraboué, F., S. Geoffroy and M. Prat, Physics of Fluids 16, 615-624 (2004)

24. Brush, D. J., and N.R. Thomson, Water Resources Research 39, 1085, doi:10.1029/2002WR001346 (2003)

5. Ge, S., Water Resours. Res. 33, 53-61. (1997)

26. Mallikamas, W. and H. Rajaram, Water Resources Research 46, W08506, doi:10.1029/2009WR008779 (2010)

27. Nicholl, M. J. and R.L. Detwiler, Geophysical Research Letters 28, 4355-4358 (2001)

28. Wang,L., M. Bayani Cardenas, D.T. Slottke, R.A. Ketcham and J.M. Sharp Jr, Water Resources Research 51, 2064-2080, doi:10.1002/2014WR015815 (2015)

29. Skjetne, E., A. Hansen and J.S. Gudmundsson, J. Fluid Mech. (1999), vol. 383, 1-28 (1999).

30. Talon L., H. Auradou and A. Hansen, Frontiers in Physics, 23 April (2014) http://dx.doi.org/10.3389/fphy.2014.00024.

31. Roustaei, A., T. Chevalier, L. Talon and I. Frigaard, Journal of Fluid Mechanics, 805, 222-261 (2016).

32. Bao K., A. Lavrov and H.M. Nilsen, Comput Geosci, 21, 13131324 (2017).

33. Yan Y., and J. Koplik, Phys. Rev. E, 77, 036315 (2008).

34. Brown S.R., J. Geophys. Res. B100, 5941-5952 (1995)

35. Gentier, S. Morphologie et comportement hydromécanique d'une fracture naturelle dans le granite sous contrainte normale, (Ph.D. Thesis, Univ. d'Orléans, France, 1986)

36. Plouraboué F., P. Kurowski, J.-P. Hulin, S. Roux and J. Schmittbuhl, Phys. Rev. E 51, 1675-1685 (1995)

37. Brown S.R., and C.H.Scholz, J. Geophys. Res. 90, 12575-12582 (1985)

38. N.O. Myers, Wear 5, 182-191 (1962)

39. Brown, S.R., R.L. Kranz and B.P. Bonner, Geophys. Res. Letters 13, 1430-1433 (1986)

40. Adler P.M., Porous Media: Geometry and Transports (Butterworth/Heinemann, Stoneham, MA, 1992)

41. Mourzenko V.V., J.-F. Thovert and P.M. Adler, Phys. Rev. E 53, 5606-5626 (1996)

42. Katz A.J. and A.H. Thompson, Phys. Rev. B 34, 9179-8181 (1986)

43. Talon L., H. Auradou and A. Hansen, Phys. Rev. E 82, 046108 (2010) 